\title{
Utilidad y costo-efectividad de los exámenes preoperatorios en cirugía otorrinolaringológica
}

\section{Usefulness and cost-efectiveness of preoperative test in otolaryngological surgery}

\author{
Carlos Namoncura $\mathrm{P}^{1}$, Hayo Breinbauer $\mathrm{K}^{2}$.
}

\begin{abstract}
RESUMEN
Introducción: La práctica de realizar exámenes preoperatorios, aunque muy difundida, carece de utilidad demostrada o de argumentos científicos que la sustenten. Más aún sus costos, pueden ser muy elevados.

objetivo: Determinar la utilidad y costo-efectividad de los exámenes preoperatorios en otorrinolaringología.

Metodología: Estudio retrospectivo de una muestra de 160 fichas clínicas de pacientes ASA I (2 a 40 años, sanos) operados en el Hospital San Juan de Dios en el año 2006.

Resultados: Ningún examen preoperatorio permitió prever una complicación perioperatoria. Dentro de los pacientes con exámenes normales se registraron 4 complicaciones. Se necesitaron 70 exámenes (con un costo de $\$ 703.500$ pesos chilenos o USD \$1,256) para detectar una patología. La detección de anormalidades, obligó al mayor uso de recursos mediante intervención clínica (repetición de exámenes o interconsultas), se asoció a un aumento del número de consultas médicas realizadas $(4,4$ vs 2,53 $p=0,0002)$ y un aumento de los días de hospitalización (2,5 vs 1,77p $=0,025)$, sin reportar ningún beneficio. Cuarenta a cincuenta por ciento de las solicitudes de nasofaringolaringoscopías, audiometrías e impedanciometrías no mostraron justificación clínica. Se repitieron $20,1 \%$ de los exámenes preoperatorios, principalmente debido a su caducidad (45,1\%). El costo oportunidad asociado a exámenes preoperatorios fue en promedio $\$ 28.608$ por paciente, 10 que anualmente equivale (como referente) al $27 \%$ del costo de todas las amigdalectomías realizadas en este centro cada año.

Discusión: El uso rutinario de exámenes preoperatorios parece ser una práctica costosa y sin utilidad.

Palabras clave: Análisis costo-beneficio, exámenes diagnósticos de rutina, procedimientos quirúrgicos otorrinolaringológicos.
\end{abstract}

\begin{abstract}
Introduction: Although most surgeons order pre-operative laboratory tests, this practice is not based upon any evidence. Furthermore, they impose a potentially important monetary cost on the patient.
\end{abstract}

Médico. Servicio de Otorrinolaringología, Hospital San Juan de Dios.

2 Médico-Cirujano, Escuela de Medicina, Universidad de Chile. 
Aim: To determine the usefulness and cost-effectiveness of preoperative tests in otolaryngology.

Material and method: Retrospective study. A sample of 160 medical records of ASA I patients (2 to 40 years of age, without other known pathologies) that underwent surgery at the San Juan de Dios Hospital during 2006 was reviewed.

Results: None of the tests ordered predicted any of the perisurgical complications found. Among patients with normal results, 4 had complications. It took 70 tests (at a cost of $\$ 703,500$ Chilean pesos or U\$D 1256) to detect a relevant pathology. Detection of abnormalities in test results forced significant more clinical interventions, and was associated with an increased number of medical consults (4.4 vs $2.53 p=0.0002)$ and a prolonged hospital stay (2.5 vs $1.77 p=0025) .20 .1 \%$ of the test were repeated tests, mainly because they had passed the valid date(45.1\%). The opportunity-cost associated with preoperative examinations averaged $\$ 28,608$ per patient, which (as a reference) is equivalent annually to $27 \%$ of the cost of all tonsillectomies performed at this center each year.

Conclusion: Routine pre-operative testing seems to be an expensive and useless practice.

Key words: Cost-Benefit Analysis, Routine Diagnostic Tests, Otorhinolaryngologic Surgical Procedures.

\section{INTRODUCCIÓN}

Realizar exámenes preoperatorios (EP) es una rutina firmemente instalada en la práctica quirúrgica. Existen diferentes razones teóricas que sustentan esta práctica:

a) Detectar anormalidades que puedan influir en el riesgo perioperatorio.

b) Establecer un parámetro basal que pueda monitorizarse y cambiar una vez que se realice el procedimiento.

c) Razones médico-legales.

Implementar protocolos preoperatorios representa un alto costo en términos monetarios, tiempo, recurso humano y días laborales perdidos. En Estados Unidos el costo de los exámenes preoperatorios se calcula en 3 mil millones de dólares anuales ${ }^{2}$. En Chile, desconocemos el costo que ellos implican.

Más aún, en pacientes sin factores de riesgo conocidos, los resultados de EP no parecen afectar significativamente la conducta quirúrgica o la evolución posoperatoria ${ }^{3}$. Esto es especialmente claro en procedimientos electivos. Por otro lado, resultados falsos-positivos en EP causan retraso en el tratamiento quirúrgico ${ }^{4}$. Así, resulta razonable realizar procedimientos quirúrgicos sin EP.
Narr y cols. reportaron procedimientos anestésicos y quirúrgicos seguros en 1.044 pacientes en quienes no se realizaron EP en los tres meses anteriores a la cirugía. Concluyeron que pacientes estudiados mediante historia clínica y examen físico, en quienes no se han determinado factores de riesgos clínicos, pueden ser sometidos a cirugía obviando la toma de EP, sin aumentar el riesgo perioperatorio ${ }^{5}$.

En un análisis retrospectivo de 3.782 pacientes clínicamente sanos, 160 tuvieron exámenes sustancialmente anormales ${ }^{6}$. La mayoría de las anormalidades no se mencionaron en las notas preoperatorias y en ningún caso cambió el desenlace de la operación o la anestesia, habiéndose 0 no instituido tratamiento. Basados en estos resultados, la Clínica Mayo (Rochester, Minnesota) no practica EP a pacientes quirúrgicos, sanos y menores de 40 años (pacientes categoría ASA 1 de riesgo preanestésico, según la Academia Americana de Anestesia).

Otra estrategia para disminuir costos relativos al uso de EP (que incluyen mayor tiempo de hospitalización y mayor frecuencia de suspensión de actos quirúrgicos), es la creación de cuestionarios estandarizados para identificar factores de riesgo (Anexo 1) 2,7-10, y evaluación clínica detallada ${ }^{11}$. Sin embargo, las prácticas deben 


\section{Anexo 1. Cuestionario preoperatorio para historia de sangrado ${ }^{17}$}

1. ¿Hay historia familiar o personal de sangrado 0 heridas fáciles?

2. ¿Tiene epistaxis, menstruación abundante 0 sangrado articular 0 muscular?

3. Tuvo sangrado después de:

a. ¿Caída de dientes o procedimiento dental?

b. ¿Cirugías previas?

c. ¿Heridas previas?

d. ¿Caída del cordón umbilical?

4. ¿Ud. 0 algún pariente ha recibido transfusiones?

5. ¿Está tomando antihistamínicos, aspirina o AINES?

6. ¿Algún familiar padece hemofilia, enfermedad de von Wllebrand, recuento de plaquetas bajo u otra patología sanguínea?

individualizarse a las necesidades de cada institución $n^{1,2,8}$

Existen numerosos artículos en la literatura médica acerca de costo-eficiencia y la relevancia de Ios EP, que no apoyan la realización rutinaria de dichos exámenes. Hasta nuestro conocimiento, no se han realizado estudios en una población chilena acerca del costo-eficiencia y relevancia médica de los exámenes preoperatorios en procedimientos otorrinolaringológicos.

El objetivo del presente trabajo, es determinar la utilidad y costo-eficiencia de los exámenes preoperatorios en cirugía otorrinolaringológica, en pacientes ASA I (con edad entre 2 a 40 años).

\section{MATERIAL Y MÉTODO}

Se realizó un estudio retrospectivo y descriptivo. De un universo total de 718 pacientes ASA I ( 2 a 40 años de edad, sanos) operados en el Servicio de Otorrinolaringología del Hospital San Juan de Dios en 2006, se seleccionó aleatoriamente una muestra de 160 casos en los que se procedió a revisión de su ficha clínica. Se excluyeron 20 por no contar con el expediente completo. Por lo tanto, se analizaron 140 fichas clínicas, representando 18,7\% del universo.

Para cada caso se registraron como variables independientes edad, género, procedimiento quirúrgico realizado y EP pedidos. Como variables dependientes se consideraron los resultados de dichos exámenes, la necesidad (y motivo) de repetirlos, número de consultas médicas preoperatorias, número de suspensiones del procedimiento quirúrgico, complicaciones peri y posoperatorias, y días de hospitalización.

Los EP fueron agrupados en 1) "Exámenes de rutina", incluyendo hemograma (por procedimiento automatizado), velocidad de sedimentación (VHS), tiempo de protrombina (TP) y tiempo de tromboplastina parcialmente activado (TTPA); y 2) "Exámenes propiamente otorrinolaringológicos (ORL)", que comprenden nasofaringolaringoscopía endoscópica (NFL), audiometría en campo silente, impedanciometría; 3) Otros exámenes preoperatorios.

Se consideró alteración de un examen cuando su resultado se encontraba fuera de los rangos referenciales del laboratorio central del Hospital San Juan de Dios.

Para exámenes $\mathrm{ORL}$ se evaluó la pertinencia de su solicitud en cada caso, de acuerdo a los datos consignados en ficha clínica y de acuerdo al criterio de los investigadores.

El costo asociado a prestación de EP y las consultas médicas necesarias para su interpretación antes de la cirugía, fueron obtenidos en el SOME (Sección de Orientación Médica y Estadísticas) del Hospital San Juan de Dios y del manual de aranceles FONASA MAI ${ }^{12}$.

Utilizando software estadístico SPSS 16.0, se realizaron análisis de frecuencias, comparación de promedios con prueba $t$ de student ${ }^{2}$ de MantelHaenszel y razón de momios (odds ratio). Además se estimó costo-efectividad en términos de costooportunidad (recursos que podrían ser utilizados con otro fin), y recursos necesarios para aumentar en una unidad el hallazgo de una alteración clínicamente significativa en los EP. 


\section{RESULTADOS}

El grupo de estudio final de 140 pacientes, consideró 72 hombres $(51,4 \%)$ y 68 mujeres $(48,6 \%)$ con una edad promedio de 10,4 años (rango entre 2 y 35 años).

La gran mayoría de las intervenciones realizadas fueron adenoidectomías y/o amigdalectomías y/o punciones timpánicas (Tabla 1). Esta distribución coincide con un estudio recientemente presentado acerca de la frecuencia de procedimientos quirúrgicos realizados en el mismo centro ${ }^{13}$, validando esta muestra.

En la Tabla 2 detallamos las características de Ios EP de rutina realizados a los pacientes de la muestra. En un quinto de los pacientes fue necesario repetir al menos uno de estos exámenes (principalmente debido a la caducidad de éstos (Tabla 3). En 10 casos (7,1\% del total) se encontró una alteración que motivara continuar con el proceso diagnóstico. Entre estos pacientes sólo 2 pacientes demostraron una entidad clínica significativa, siendo en ambos casos una enfermedad de von Willebrand, lo que motivó evaluación por hematólogo y preparación preoperatoria con crioprecipitado. No hubo complicaciones perioperatorias.

En la Tabla 3 se aprecia que la principal causa de repetición de exámenes fue la caducidad, ninguno de los exámenes de rutina repetidos por este motivo (o cualquiera otra causa) encontró alteraciones.

Con respecto a los exámenes propiamente $\mathrm{ORL}$, observamos en la Tabla 4 como en casi dos tercios de los pacientes de la muestra en los que se tomó una audiometría o impedanciometría inicial, ésta se repitió. Más aún, en más de un cuarto de estos casos estos exámenes se volvieron a realizar una tercera vez. La gran mayoría de estas situaciones fue secundaria a la caducidad de los EP audiológicos, 0 por controles programados a la espera del procedimiento quirúrgico (Tabla 3). Al analizar la pertinencia clínica de la solicitud de estos exámenes considerando el impacto que éste tendría sobre cambios en el manejo de estos pacientes, encontramos algo más de la mitad de las audiometrías, impedanciometrías y NFL tendrían justificación. En

Tabla 1. Distribución de cirugías

\begin{tabular}{|lcc|}
\hline & $\mathrm{n}$ & $\%$ \\
\hline Adenoidectomías y/o amigdalectomías y/o PAT & 116 & 82,8 \\
Miringoplastías/timpanoplastías & 11 & 7,8 \\
Rino y/o septoplastías & 10 & 7,1 \\
Otros & 3 & 2,1 \\
\hline
\end{tabular}

Tabla 2. Exámenes preoperatorios de rutina

\begin{tabular}{|c|c|c|c|c|}
\hline $\begin{array}{l}\text { Exámenes } \\
\text { de rutina }\end{array}$ & 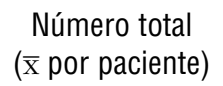 & Repeticiones* & $\begin{array}{l}\text { Alteraciones } \\
\text { encontradas }\end{array}$ & $\begin{array}{l}\text { Patología } \\
\text { confirmada** }\end{array}$ \\
\hline Hemograma & $148(1,06)$ & $6 \%$ & $1(0,7 \%)$ & 0 \\
\hline VHS & $147(1,05)$ & $5 \%$ & 0 & 0 \\
\hline TP & $154(1,10)$ & $10 \%$ & $3(2,1 \%)$ & 0 \\
\hline TTPA & $158(1,13)$ & $12 \%$ & $6(4,3 \%)$ & 2 (von Willebrand) \\
\hline Total & $607(4,33)$ & $\underset{* \star *}{20,7 \%}$ & $10 \underset{\star \star \star \star \star}{(7,1 \%)}$ & $2(1,4 \%)$ \\
\hline
\end{tabular}

*Porcentaje de pacientes a los que se repitió el examen. **Patología confirmada por exámenes complementarios y especialista. ${ }^{* *}$ Porcentaje del total de pacientes que requirió la repetición de al menos un examen. ${ }^{* \star \star *}$ Porcentaje del total de pacientes que mostró alguna alteración en al menos uno de sus exámenes. 
Tabla 3. Motivos de repetición de exámenes

\begin{tabular}{|lcc|}
\hline Motivo & $\mathrm{n}$ & $\%$ \\
\hline Caducidad $^{\star}$ & 69 & 45,1 \\
Alteración de exámenes iniciales $_{\text {Controles programados u otras causas ** }}^{*}$ & 50 & 32,5 \\
Total exámenes repetidos $^{*}$ & 34 & 22,5 \\
\end{tabular}

Se incluyen en esta tabla exámenes de rutina y propiamente $\mathrm{ORL}$.

*Definido arbitrariamente 6 meses de duración. ${ }^{* *}$ En caso de controles audiométricos programados 0 duda clínica de diagnóstico o indicación quirúrgica.

Tabla 4. Exámenes preoperatorios propiamente otorrinolaringológicos

\begin{tabular}{|lcccc|}
\hline Examen ORL & $\begin{array}{c}\text { Número total } \\
(\bar{x} \text { por paciente })\end{array}$ & $\begin{array}{c}\text { Una } \\
\text { repetición* }\end{array}$ & $\begin{array}{c}\text { Dos } \\
\text { repeticiones * * }\end{array}$ & $\begin{array}{c}\text { Pertinencia } \\
\text { del examen } \\
\text { solicitado }\end{array}$ \\
\hline Nasofaringolaringoscopía & $15(0,11)$ & $0 \%$ & $0 \%$ & $60 \%$ \\
Audiometría & $36(0,26)$ & $63,2 \%$ & $26,3 \%$ & $52,8 \%$ \\
Impedanciometría & $34(0,24)$ & $70,6 \%$ & $29,4 \%$ & $50 \%$ \\
Total & 85 & & & \\
\hline
\end{tabular}

*Porcentaje de pacientes a los que se repitió el examen al menos una vez. ${ }^{*}$ Porcentaje de pacientes a quienes se les realizó el examen una tercera vez.

el porcentaje restante no se encontró algún fundamento clínico explícito en la ficha clínica que avalara la decisión de repetir el examen.

Con respecto al resultado de los exámenes de rutina, podemos ver en la Tabla 5, como fueron necesarios 70 exámenes de rutina (con un costo de $\$ 703.500$ pesos chilenos) para encontrar una alteración clínicamente relevante. Esto, permitió detectar 2 casos de enfermedad de von Willebrand, Io que modificó su manejo como ya se expuso.

Ningún EP alterado se asoció a alguna complicación perioperatoria.

Más aún, dentro de los casos inicialmente determinados sin mayor riesgo (con exámenes preoperatorios normales) hubo 4 complicaciones (2,9\% del total de la muestra), todos ellos sangrados posoperatorios en adeno-amigdalectomías.

No es posible calcular razón de momios (odds ratio) para la comparación de complicaciones según alteración de EP debido a la ausencia de casos en alguna de las categorías.
Al evaluar en conjunto los casos donde se necesitó una acción médica, y las complicaciones observadas, como resultados que demandaron un cambio en el manejo del paciente, observamos una razón de momios de 7,87 con un intervalo de confianza sobre 1, y un valor de $\chi^{2}$ asociado a un $p<0,05$. Esto podría reflejar, que los EP obligan a intervenir con más frecuencia en casos con resultados alterados en comparación a pacientes sin alteración en sus exámenes, sin embargo, sin impacto en la tasa de complicaciones.

Por otro lado, aunque los EP no ayudaron a prever complicaciones, sí se asociaron significativamente a mayor número de consultas médicas, mayor número de días de hospitalización (principalmente debido a la necesidad de repetir exámenes y visitas anestésicas).

En términos de costo-efectividad, se necesitaron 70 exámenes de rutina para encontrar una patología que motivó un cambio de conducta dentro del manejo del paciente (2 casos de EvW). 
Tabla 5. Exámenes alterados y resultados

\begin{tabular}{|c|c|c|c|}
\hline & $\begin{array}{l}\text { Exámenes } \\
\text { alterados }\end{array}$ & $\begin{array}{l}\text { Exámenes } \\
\text { normales }\end{array}$ & Significancia estadística \\
\hline $\begin{array}{l}\text { Número } \\
\text { (\% del total) }\end{array}$ & $\begin{array}{c}10 \\
(7,1 \%)\end{array}$ & $\begin{array}{c}130 \\
(92,8 \%)\end{array}$ & \\
\hline $\begin{array}{l}\text { Patología confirmada que requirió } \\
\text { acción médica (\% del total) }\end{array}$ & $\begin{array}{c}2 \\
(1,4 \%)\end{array}$ & $\begin{array}{c}0 \\
(0 \%)\end{array}$ & $\begin{array}{l}\text { - Se requieren } 70 \text { exámenes } \\
\text { de rutina para encontrar } 1 \\
\text { patología. } \\
\text { - Ninguna alteración en } \\
\text { exámenes preoperatorios } \\
\text { asoció una complicación }\end{array}$ \\
\hline $\begin{array}{l}\text { Complicaciones perioperatorias } \\
\text { ( } \% \text { del total) }\end{array}$ & $\begin{array}{c}0 \\
(0 \%)\end{array}$ & $\begin{array}{c}4 \\
(2,9 \%)\end{array}$ & \\
\hline $\begin{array}{l}\text { Total de situaciones con repercusión } \\
\text { en el manejo** }\end{array}$ & $\begin{array}{c}2 \\
(1,4 \%)\end{array}$ & $\begin{array}{c}4 \\
(2,9 \%)\end{array}$ & $\begin{array}{l}\text { Odds Ratio }=7,87 \\
(1,25-49,67)^{\star * *} \\
2^{2}=7,095 ; p<0,008\end{array}$ \\
\hline $\begin{array}{l}\text { Suspensiones de la cirugía } \\
\text { (\% por paciente } \pm \text { desv. estándar) }\end{array}$ & $\begin{array}{c}0,78 \\
(\mathrm{DE} \pm 0,92)\end{array}$ & $\begin{array}{c}1,1 \\
(\mathrm{DE} \pm 0,876)\end{array}$ & $\begin{array}{l}\text { p-value }=0,284 \\
\text { (t-student) }\end{array}$ \\
\hline $\begin{array}{l}\text { Número de consultas médicas } \\
\text { (x por paciente } \pm \text { desv. estándar) }\end{array}$ & $\begin{array}{c}4,4 \\
(\mathrm{DE} \pm 1,17)\end{array}$ & $\begin{array}{c}2,53 \\
(\mathrm{DE} \pm 0,97)\end{array}$ & $\begin{array}{l}{ }^{*} p \text {-value }=0,0002 \\
\text { (t-student) }\end{array}$ \\
\hline $\begin{array}{l}\text { Días de hospitalización } \\
\text { (\% por paciente } \pm \text { desv. estándar) }\end{array}$ & $\begin{array}{c}2,5 \\
(\mathrm{DE} \pm 3,37)\end{array}$ & $\begin{array}{c}1,77 \\
(\mathrm{DE} \pm 0,49)\end{array}$ & $\begin{array}{l}{ }^{*} p \text {-value }=0,025 \\
\text { (t-student) }\end{array}$ \\
\hline
\end{tabular}

*Estadísticamente significativo. * ${ }^{\star}$ Para el análisis de Odds Ratio y $\chi^{2}$ se consideraron en conjunto las patologías que requirieron acción médica y las complicaciones como eventos que cambiaron el manejo del paciente y cuyo reconocimiento era el objetivo del examen preoperatorio. ${ }^{* \star \star}$ Valor de Odds Ratio e intervalo con $95 \%$ de confianza.

El costo-oportunidad (definido como los recursos ocupados en una tarea que podrían ser invertidos en otro servicio) de realizar EP de rutina (incluyendo el costo del examen en sí mismo y el aumento en número de consultas y días de hospitalización que se asocia a su interpretación y repeticiones) es de $\$ 28.608,29$ pesos chilenos en promedio por paciente operado. Extrapolado a la totalidad de pacientes ASA I operados anualmente en el Servicio de Otorrinolaringología del Hospital San Juan de Dios (718 pacientes en 2006), el costo-oportunidad anual de los exámenes de rutina alcanza los $\$ 20.540 .748,12$ pesos chilenos, lo que equivale a los costos asociados a realizar 162 amigdalectomías con 0 sin adenoidectomías, con sus días de hospitalización y consultas médicas correspondientes contabilizadas (este número corresponde al $27 \%$ de todas las amigdalectomías realizadas en un año en este centro).

El costo-oportunidad asociado a la realización de nasofaringolaringoscopías (NFL) no pertinen- tes es de $\$ 963$ chilenos por paciente, que extrapolado al total de operados anuales alcanza $\$ 134.880$ chilenos. El costo-oportunidad asociado a la realización de audiometrías e impedanciometrías no pertinentes es de $\$ 12.814$ por paciente, que extrapolado equivale a $\$ 461.320$ anuales.

\section{DISCUSIÓN}

Se suelen solicitar exámenes preoperatorios a todos los pacientes que serán sometidos a cirugía, sin embargo, las razones solamente tienen fundamentos teóricos o legales. En Chile, la Sociedad de Anestesiología sugiere: "La solicitud de exámenes complementarios no debiera ser un acto médico de rutina, sino que éstos debieran solicitarse según los hallazgos de la historia, el examen físico y las características de la cirugía que se va a realizar"14. 
Esta postura también es adoptada por la Academia Americana de Otorrinolaringología y Cirugía de Cabeza y Cuello ${ }^{15}$ y la Agencia Nacional de Acreditación y de Evaluación en Salud en Francia ${ }^{16}$.

En nuestro trabajo los EP de rutina, en pacientes ASA I, tuvieron baja utilidad en cuanto a su impacto en el manejo clínico de los pacientes, se requirió de un elevado número de exámenes al azar para detectar una patología que ameritara cambios en la conducta. Los exámenes que con mayor frecuencia presentaron alteraciones fueron en relación a la hemostasia, sin embargo se ha descrito que los tiempos de coagulación no predicen la presentación de hemorragia perioperatoria ${ }^{14}$. En nuestra serie, los casos en los que se llegó al diagnóstico de enfermedad de von Willebrand, la patología de la hemostasia más frecuente, su determinación sí influyó en el manejo del paciente y determinó una estadía más prolongada y mayores costos, justificados en un mayor riesgo de sangrado, motivo por el cual los costos no se incluyeron en el cálculo de costo-eficiencia.

Este resultado, en particular, estimula a realizar un estudio controlado, con pacientes a los que se realicen exámenes de rutina (grupo control) y a quienes no se les realicen (grupo experimental), comparando la tasa de complicaciones reales que existan entre ambos grupos, y no sólo la necesidad teórica-clínica de realizar una intervención. Experiencias internacionales apoyan dicho análisis, no habiendo encontrado diferencias estadísticas entre estos grupos s., $^{2,7,9,10}$, especialmente si se realiza tamizaje clínico previo con encuestas como la expuesta en el Anexo $1^{16}$. En nuestro contexto nacional, el presente trabajo justifica y motiva la realización de tal estudio.

Tampoco existe un fundamento científico para respaldar la premisa de caducidad de los EP si no ha habido ningún cambio en el estado clínico del paciente. Incluso, se ha mencionado que solamente $0,4 \%$ de los EP repetidos al momento de la cirugía serían anormales ${ }^{18}$. En otros estudios tampoco se ha encontrado que los exámenes preoperatorios cambien significativamente si no ha habido una diferencia en el estado clínico de los pacientes al menos durante cuatro meses ${ }^{19}$.

En nuestra revisión no hubo ningún examen caducado que haya cambiado sus resultados. Esto resulta de alto impacto si consideramos que 22,1\% de los exámenes realizados son por repetición.
Otro aspecto importante se refiere al control de la pertinencia clínica de la realización de exámenes. Casi la mitad de las indicaciones de NFL, audiometrías e impedanciometrías carecían de cualquier propósito razonable, más allá de la indicación avalada por la duda. Es tarea de cada médico el cuestionarse la necesidad de realizar un examen 0 no, considerando el costo acumulativo de ellos. El costo anual asociado a los exámenes de rutina superó en casi 40 veces al mal uso de exámenes otorrinolaringológicos. Así, una medida de mayor impacto sería el transferir el costooportunidad de los exámenes de rutina hacia una prestación de mayor utilidad.

Cabe considerar que el costo-oportunidad calculado, deja de lado el análisis de factores de más difícil objetivación, como es el costo social. En promedio, cada paciente pierde 2,49 días laborales debido a la realización de exámenes de rutina (sea por consulta médica o día de hospitalización). Al extrapolarlo a la población operada anualmente, alcanza a la pérdida de 1.785 días laborales anuales (un año de trabajo para 7,4 personas) sólo por motivo de exámenes de rutina para cirugía otorrinolaringológica en el Hospital San Juan de Dios.

Queremos constatar algunos sesgos en este estudio, la mayoría secundarios a su diseño retrospectivo, se debió excluir a 20 de los pacientes elegibles por falta de registros completos (12,5\%), y no se pudo calcular la incidencia real de eventos. Además no se contó con información sobre pacientes en los que se decidió no realizar la cirugía en forma permanente, y el impacto de exámenes preoperatorios en dicha decisión. Lo anterior invita a realizar un nuevo estudio prospectivo del tema.

En síntesis, realizar EP rutinarios en pacientes clínicamente sanos es una práctica cuestionable. Diferentes estudios evalúan la utilidad de cuestionarios estandarizados basados en datos clínicos relevantes, los cuales han eliminado la necesidad de EP de rutina. Ésta parece ser una política más racional que la tradicional, en la cual se solicitan EP independientemente del estado de salud del paciente.

La limitación de los EP puede significar un ahorro sustancial para las instituciones de salud. Salvo, los 2 casos detectados de enfermedad de von Willebrand, las anormalidades en los exámenes preoperatorios no se asociaron a incremento en la frecuencia de complicaciones perioperatorias 
o en el tiempo de estadía hospitalaria. El análisis de costo-efectividad mostró que los exámenes preoperatorios en pacientes adultos jóvenes clínicamente sanos es una práctica costosa y poco eficiente.

\section{BIBLIOGRAFÍA}

1. Smetana GW, Macpherson DS. The case against routine preoperative laboratory testing. Med Clin N Am 2003; 87: 7-40.

2. FISCHER SP. Cost-effective preoperative evaluation and testing. Chest 1999; 115: 96-100.

3. Morales C, Mata M, Cárdenas LE. Costo-beneficio de los exámenes preoperatorios de rutina en cirugía electiva. Cir Ciruj 2005; 73: 25-30.

4. RoIzen MF. Cost-effective preoperative laboratory testing. JAMA 1994; 271: 319-20.

5. Narr BJ, Warner ME, Schroeder SR, Warner MA. Outcomes of patients with no laboratory assessment before anesthesia and a surgical procedure. Mayo Clin Proc 1997; 72: 505-9.

6. Narr BJ, Hansen TR, Warner MA. Preoperative laboratory screening in healthy Mayo patients: cost-effective elimination of tests and unchanged outcomes. Mayo Clin Proc 1991; 66: 155-9.

7. Gathe-Ghermay JC, LIU LL. Preoperative programs in anesthesiology. Anesthiol Clin N Am 1999; 17: 335-53.

8. Van Klel WA, Moons KGM, Rutten Clg, Schuurhuis A, Knape JTA, Kalkman C, Grobbee D. The effect of outpatient preoperative evaluation of hospital in patients on cancellation of surgery and length of hospital stay. Anesth Analg 2002; 94: 644-9.

9. Roizen MF, Coalson D, Hayward RS, Schmittiner J, Thisted RA, Apfellbaum Jl, Stocking CV, Cassel CK, Pompel P, Ford DE, Steinberg EP. Can patients use an automated questionnaire to define their current health status? Med Care 1992; 30: MS74-84.

10. Tremper KK. Paper "Preoperative computer". Anesthesiology 2000; 92: 1212.
11. Roizen MF, Kaplan EB, Sheiner LB, et al. Elimination of unnecessary laboratory tests by preoperative questionnaire [abstract]. Anesthesiology 1984; 61: A455.

12. Fondo Nacional de Salud. Arancel Modalidad Atención Institucional. Disponible en www.fonasa.cl [Consultado el 20 de marzo de 2009].

13. Breinbauer $H$, Castillo $C$, Arancibia M. Análisis epidemiológico en 12 años de cirugía otorrinolaringológica en el hospital San Juan de Dios. Presentado en el LXV Congreso Chileno de Otorrinolaringología y Cirugía de Cabeza y CueIlo, 2 al 6 de diciembre 2008, Pucón, Chile.

14. Sociedad de Anestesiología de Chile. Recomendaciones en la evaluación preoperatoria pediátrica. Disponible en: http:// www.sachile.cl/medicos/recomendaciones/ pre_anestesica/evaluacion_nino.php [Consultado el 20 de marzo de 2009].

15. Asaf T, Reuveni H, Yermiahu T et al. The need for routine pre-operative coagulation screening tests (prothrombin time PT/partial thromboplastin time PTT) for healthy children undergoing elective tonsillectomy and/or adenoidectomy. Int $\mathrm{J}$ Ped Otorhinolaryngol 2001; 61: 217-22.

16. Agence Nationale d'Accreditation et d'Évaluation en Santé, Service des recommandations et références professionnelles. Les examens préopératoires systématiques. Paris, Diciembre 1998.

17. DeRkay CS. A Cost-effective Approach for Preoperative Hemostatic Assessment in Children Undergoing Adenotonsillectomy. Arch Otolaryngol Head Neck Surg 2000; 126: 688.

18. Macpherson DS, Snow R, Lofgren RP. Preoperative screening: value of previous tests. Ann Intern Med 1990; 113: 969-73.

19. Shah SA, Sajid SA, Asif M, et al. Significance and cost effectiveness of pre-operative routine laboratory investigations in young healthy patients undergoing elective ear, Nose \& throat Surgery. J Ayub Med Coll Abbottabad 2007; 19(2): 3-6. 